\title{
LOS PRINCIPIOS DE PRIMACÍA Y COMPLEMENTARIEDAD. UNA NECESARIA CONCILIACIÓN ENTRE LAS COMPETENCIAS DE LOS ÓRGANOS PENALES NACIONALES Y LOS INTERNACIONALES*
}

\author{
Raúl Carnevali Rodríguez**
}

\begin{abstract}
RESUMEN
Tratándose de crimenes internacionales resulta fundamental precisar las competencias entre los tribunales nacionales e internacionales. Para este efecto se han estructurado dos grandes principios, a saber, el de primacía y el de complementariedad. A fin de comprender su alcance, se examina lo acontecido con el Tribunal Militar Internacional -los llamados juicios de Nuremberg-y los tribunales penales ad hoc-para la ex Yugoslavia y Rwanda-. Asimismo, se analizan las razones que llevaron a implementar el principio de complementariedad respecto de la Corte Penal Internacional, rechazándose la idea de su primacía sobre los tribunales nacionales.
\end{abstract}

\section{CORTE PENAL INTERNACIONAL - DERECHO PENAL - JURISDICCIÓN INTERNACIONAL}

\section{The principles of primacy and complementarity. A necessary conciliation between national and international criminal authorities' powers}

\section{Abstract}

As they are international crimes, it is fundamental to specify the powers of international and national courts. For this matter, two principles have been structured, both primacy and complementarity. To be able to understand its reach, this work examines what occurred with the International Military Court - the Nuremberg Trials-and the ad hoc criminal courts -for ex Yugoslavia and Rwanda-. At the same time, the reasons for implementing a complementarity principle in the International Criminal Court are examined, rejecting the idea of its primacy over national courts.

\section{INTERNATIONAL CRIMINAL COURT - CRIMINAL LAW - INTERNATIONAL JURISDICTION}

\footnotetext{
* Este trabajo ha sido realizado dentro del marco del Proyecto Fondecyt $\mathrm{N}^{\circ} 1080060$ titulado "El Principio de complementariedad: un cambio de paradigma en la justicia internacional".

** Abogado, Doctor en Derecho, Profesor asociado de Derecho Penal y subdirector del Centro de Estudios de Derecho Penal de la Universidad de Talca, Chile. rcarnevali@utalca.cl.

Artículo recibido el $1^{\circ}$ de diciembre de 2009 y aceptado para su publicación por el Comité Editorial el 28 de mayo de 2010.
} 


\section{INTRODUCCIÓN}

$\mathrm{U}$ na de las mayores dificultades que se presentan en la persecución de determinados crímenes internacionales es cómo coordinar las competencias de los tribunales nacionales con los internacionales. En efecto, en aquellos casos en que existen competencias concurrentes (concurrent jurisdictions) es preciso disponer de reglas que diluciden cuál de ellas ha de conocer el caso en cuestión. Tarea, por cierto, nada fácil, pues entran en juego una serie de intereses, muchas veces contrapuestos, que complican su resolución. Precisamente, los estatutos de los tribunales penales internaciones ad hoc -así, para la ex Yugoslavia y Rwanda- y la Corte Penal Internacional se hacen cargo de esta cuestión, disponiendo de ciertas reglas dirigidas a precisar la esfera competencial. Tratándose de los primeros, tienen primacía sobre las instituciones nacionales, no así, en cambio, la Corte Penal Internacional, en donde el principio de complementariedad ordena que sean los Estados los llamados a resolver y juzgar ciertos crímenes de carácter internacional. La Corte Penal Internacional sólo actuará cuando los Estados no puedan o no desean hacerlo -art. 17 del Estatuto de Roma-.

En forma muy sucinta se ha expuesto cuáles son los mecanismos que hoy se conocen para resolver los problemas que presentan las competencias concurrentes, a saber, la primacía y la complementariedad ${ }^{1}$. En todo caso, la concreción de estos principios ha dado lugar a largas discusiones, pues de algún modo suponen limitar la soberanía de los Estados en el ejercicio de la actividad punitiva. Basta tener en consideración los debates suscitados hasta llegar al Estatuto de Roma, acerca de si la Corte Penal Internacional podía o no tener primacía sobre los Estados nacionales ${ }^{2}$.

A fin de graficar los problemas que en este orden se presentan, citaré como ejemplo un caso hipotético que puede ser conocido y juzgado por diversos Estados y también por el Tribunal Penal Internacional para la ex Yugoslavia $^{3}$ : ciudadano croata (jurisdicción de nacionalidad de Croacia) comete crímenes de guerra en Bosnia y Herzegovina (jurisdicción territorial bosnia) contra prisioneros de guerra que eran ciudadanos de Yugoslavia (personalidad pasiva de Yugoslavia). Este ciudadano croata se traslada como inmigrante a Austria (jurisdicción universal de Austria). Todos los Estados citados pueden legítimamente reclamar su competencia, a la que debe agregarse la del Tribunal Penal Internacional ad hoc que, conforme al art. 9 del Estatuto, dispondría de primacía sobre todos ellos.

El trabajo se centrará en el principio de primacía y complementariedad, y su alcance respecto de aquellos crímenes de derecho internacional (crimes under international law)

${ }^{1}$ Cassese, A., International Criminal Law, University Press, $2^{\text {a }}$ ed. Oxford, 2008, p. 336.

${ }^{2}$ Brown, B. "Primacy or Complementarity: Reconciling the Jurisdiction of National Courts and International Criminal Tribunals”. The Yale Journal of International Law, Vol. 23, 1998, p. 416 y ss.; El Zeidy, M.M., The principle of Complementarity in International Criminal Law, Nijhoff Publishers, Leiden-Boston, 2008, p. 126 y ss.; Stigen, J., The relationship between the International Criminal Court and National Jurisdictions. The Principle of Complementarity. Nijhoff Publishers, Leiden-Boston, 2008, p. 64 y ss.

${ }^{3}$ Expuesto en Brown, "Primacy or Complementarity", cit. nota n. 2, p. 392. 
que pueden estar sujetos a competencias concurrentes entre instituciones jurisdiccionales nacionales y tribunales internacionales. En consecuencia, no me dirigiré hacia aquellos delitos denominados crímenes de trascendencia internacional, sobre los cuales el derecho internacional impone a los Estados la obligación de implementarlos en su derecho interno; es decir, son los Estados los llamados a resolver los casos que pueden suscitarse y en donde, eventualmente, podría presentarse un conflicto de competencias entre Estados. En esta esfera no intervienen organismos internacionales ${ }^{4}$. Para estos casos, es decir, crímenes de trascendencia internacional, el derecho internacional no dispone de reglas que permitan resolver cuáles competencias prevalecen ${ }^{5}$.

Podría decirse que ese era el panorama que también se presentaba respecto de los crímenes de derecho internacional hasta los años noventa del pasado siglo ${ }^{6}$. Al menos de manera más o menos evidente, pues ya los juicios de Nuremberg -como se verá- comenzaron a sentar las bases de los futuros tribunales penales internacionales ${ }^{7}$. Hoy, en cambio, se manifiesta un nuevo equilibrio en donde se procura conciliar la competencia de los tribunales nacionales con la de los internacionales. A continuación examinaré cuál ha sido el camino recorrido y bajo qué condiciones es posible aquello.

\section{NuREMbERG y SUS CONSECUENCIAS}

Si bien existía el consenso de juzgar a los criminales nazis, a fin de evitar la impunidad que tuvo lugar tras la Primera Guerra Mundial, al menos de los jerarcas ${ }^{8}$, las

${ }^{4}$ Para mayor detalle, Matus Acuña, J.P., "La política criminal de los tratados internacionales", en Carnevali, R., (Coord.). Cuestiones de Política Criminal en los tiempos actuales. Editorial Jurídica, Santiago, 2009, p. 107 y ss.; Quintano Ripollés, A., Tratado de Derecho penal internacional e internacional penal. T. I. Madrid, 1955, p. 269 y ss. y p. 377 y ss., distinguía entre delitos de orden interno con trascendencia internacional y delitos internacionales propiamente dichos. Entre los primeros comprendía los atentados contra la soberanía estatal extranjera y la piratería. En los segundos, el genocidio y los crímenes contra la paz, por citar algunos.

${ }^{5}$ Cassese, International, cit. nota n. 1, p. 339; Brown, "Primacy", cit. nota n. 2, p. 392.

${ }^{6}$ Así se puede apreciar en Quintano Ripollés, Tratado, T. II, cit. nota n. 4, passim. Por otra parte, el caso Pinochet es un ejemplo paradigmático de los conflictos de competencias que en este orden se pueden presentar. Al respecto, Ambos, K., La nueva justicia penal internacional. Fundación Myrna Mack, Guatemala, 2000 , p. 165 y ss.

${ }^{7}$ Cassese, A., "Opinion: The International Criminal Tribunal for the Former Yugoslavia and Human Rights", en European Human Rights Law Review, 1997, p. 329 y ss.; Tiefenbrun, S.W., "The paradox of International adjudication: developments in the International Criminal Court for the Former Yugoslavia and Rwanda, the World Court and the International Criminal Court", en North Carolina Journal of International Law and commercial regulation, $\mathrm{N}^{\circ}$ 3, 2000 , p. 552 y ss.; Carnevali, R., "Hacia la conformación de un Tribunal penal internacional. Evolución histórica y desafíos futuros", en Revista de Derecho de la Universidad Católica del Norte. Sede Coquimbo. No 10, 2003, p. 34-38.

${ }^{8}$ Hay que considerar que el art. 227 del Tratado de Versalles disponía el establecimiento de un Tribunal especial para juzgar al emperador Guillermo II por ofensas supremas a la moral internacional y a la autoridad sagrada de los tratados. Sin embargo, nunca se pudo juzgar al Kaiser, pues los Países Bajos -donde se encontraba- se negaron a extraditarlo, como también a otros importantes jerarcas alemanes. Aunque el 
discrepancias se planteaban en cuanto al procedimiento. En efecto, en un principio algunos sostenían -Cámara de los Lores en 1942- que cada Estado debía ejercer sus potestades punitivas. Sin embargo, acabaron por imponerse las posturas de quienes argumentaban la necesidad de un tribunal internacional ad hoc "así, los Estados Unidos"”.

Precisamente en la Declaración de Moscú de $1943^{10}$ tres de las potencias aliadas -Estados Unidos, Gran Bretaña y la Unión Soviética- dispusieron que los criminales de guerra alemanes debían ser juzgados y sancionados por aquellos países donde fueron cometidos tales crímenes. Se seguía así el principio de territorialidad. Sin embargo, tratándose de aquellos criminales con mayor responsabilidad y sin que sus crimenes tuvieren una particular localización geográfica, debían ser juzgados conforme a reglas y procedimientos que aún no estaban definidas ${ }^{11}$. De esta forma se conformaron las bases del futuro Tribunal Militar Internacional que sesionaría en Nuremberg. Si bien se reconoció el papel de los tribunales penales nacionales, también se dispuso que debía ser otra la instancia que se hiciera cargo de los mayores criminales.

Justamente, el Estatuto de Londres de 8 de agosto de $1945^{12}$ concretó dicha Declaración, estableciendo en su preámbulo lo que sigue:

“(...) determinó que los oficiales y soldados alemanes y los miembros del partido nazi que son responsables de atrocidades y crímenes o que hayan permitido que se cometieran los mismos, serán devueltos a los países en los cuales sus abominables fechorías fueron cometidas, a fin de que puedan ser juzgados y castigados de acuerdo con las respectivas leyes de los países liberados y de los gobiernos libres que en ellos se establezcan; y considerando que dicha Declaración estipuló que no era aplicable a los criminales principales cuyas ofensas no estuvieren circunscritas a determinadas regiones y que estuvieren circunstancias a determinadas regiones y que éstos serían castigados por la decisión conjunta de los gobiernos aliados”.

\footnotetext{
Tribunal imperial (Reichsgericht) juzgó crímenes de guerra cometidos por alemanes, no tuvo mayor repercusión. Con detalle, Meron, T., "Reflections on the prosecution of war crimes by International Tribunals", en American Journal of International Law, Vol. 100, 2006, p. 553 y ss.; Además, Quintano Ripollés, Tratado, T. I, cit. nota n. 4, p. 401.

${ }^{9}$ Sobre la necesidad de un Tribunal internacional, Glueck, S., "By what Tribunal shall war offenders be tried?”, en Harvard Law Review. Vol. 56, 1942-1943, p. 1059 y ss. Comenta el trabajo de Glueck, Jiménez de Asúa, L., Tratado de Derecho penal. T. II. $5^{a}$ ed. Losada, Buenos Aires, 1992, p. 1222-1228.

${ }^{10}$ La Declaración de Moscú en http://avalon.law.yale.edu/wwii/moscow.asp (consultado el 5 de noviembre de 2009).

${ }^{11}$ El Zeidy, The principle, cit. nota n. 2, p. 74.

${ }^{12}$ Estatuto de Londres en http://avalon.law.yale.edu/imt/imtchart.asp (consultado el 5 de noviembre de 2009). También en Quintano Ripollés, Tratado, T. I, cit. nota n. 4, p. 406-408. El texto de constitución del Tribunal Militar Internacional en http://avalon.law.yale.edu/imt/imtconst.asp (consultado el 5 de noviembre de 2009). También en Quintano Ripollés, Tratado, T. I, cit. nota n. 4, p. 408-415.
} 
En su art. 1 ordenó lo siguiente:

"Será establecido, después de consulta con el Consejo de Control de Alemania, un Tribunal Militar Internacional para el enjuiciamiento de los criminales de guerra cuyos crímenes no tuvieren localización geográfica particular, y que sean acusados individualmente o en su calidad de miembros de organizaciones o grupos, o en ambas condiciones".

Por su parte el art. 6 del Estatuto dispuso lo que sigue:

"Nada de lo dispuesto en este Acuerdo afectará a la jurisdicción o a los poderes de ningún tribunal nacional ni de los tribunales de ocupación ya establecidos o que establecieren en cualquier territorio aliado o en Alemania para enjuiciar a los criminales de guerra".

Pues bien, una primera aproximación nos sugiere que se dispuso de competencias concurrentes entre los tribunales nacionales y otras cortes con el Tribunal Militar Internacional. Sin embargo, considerando cuestiones relativas a ratione loci-crímenes no tuvieren localización geográfica particular- y ratione personae -criminales principales- se aplicó el principio de primacía a favor del Tribunal Militar Internacional ${ }^{13} \mathrm{o}$, como algunos han sostenido, en estos casos particulares primó la supremacía del derecho internacional por sobre derecho nacional ${ }^{14}$. Es decir, reuniéndose los presupuestos ya indicados sólo tenía competencia el Tribunal Militar Internacional. A lo anterior, se debe tener presente que el Consejo de Control Aliado a través de la Ley N $\mathrm{N}^{\circ} 10$ de 20 de diciembre de $1945^{15}$ estableció una base legislativa común para todas las zonas de ocupación en Alemania - "Castigo para las personas culpables de crímenes de guerra, crímenes contra la paz y crímenes contra la humanidad”-. De esta Ley también se desprendía la supremacía del Tribunal Militar Internacional sobre las otros tribunales nacionales.

Aun cuando los juicios de Nuremberg ${ }^{16}$ han sido objeto de críticas, algunas discutibles por cierto ${ }^{17}$, lo incuestionable es que planteó la necesidad de disponer de mecanismos

${ }^{13}$ Kleffner, J.K., Complementarity in the Rome Statute and National Criminal Jurisdictions. University Press, Oxford, 2008, p. 63.

${ }^{14}$ El Zeidy, The principle, cit. nota n. 2, p. 75.

${ }^{15}$ Ley $\mathrm{N}^{\mathrm{o}} 10$ de 20 de diciembre de 1945 en http://avalon.law.yale.edu/imt/imt10.asp (consultado el 5 de noviembre de 2009).

16 Ver el texto completo de la sentencia en American Journal of International Law, Vol. 41, 1947, p. 172 y ss. También en http://avalon.law.yale.edu/subject_menus/judcont.asp (consultado el 5 de noviembre de 2009). Respecto al Tribunal Militar Internacional del Extremo Oriente, constituido en Tokio, cfr. Quintano Ripollés, Tratado, T. I, cit. nota n. 4, p. 433 y ss.; Jiménez de Asúa, Tratado, T. II, cit. nota n. 9, p. 1215-1217.

${ }^{17}$ Interesantes resultan las observaciones y críticas planteadas por H. Donnedieu de Vabres, quien fue juez en representación de Francia, en "Le procès de Nuremberg”, en Revue de Science Criminelle et de Droit Pénal Comparé, $\mathrm{N}^{\circ} 2$, 1947, p. 171 y ss.; también debe considerarse la muy ilustrada exposición de Jiménez 
más efectivos para juzgar crímenes internacionales, entre ellos el de instaurar una Corte permanente $^{18}$. Asimismo, la importancia de regular las competencias de los tribunales nacionales e internacionales y cómo debían relacionarse. Es cierto que pasaron largos años para llegar a la Corte Penal Internacional, pero el desafío ya estaba sobre la mesa.

\section{Los Tribunales Penales Internacionales para la EX Yugoslavia y Rwanda}

Tras los procesos de Nuremberg y Tokio las Naciones Unidas -se conformó para ello una Comisión de Derecho Internacional- comenzó a trabajar en la elaboración de un Proyecto de Código de crímenes contra la paz y la seguridad de la humanidad ${ }^{19}$. Asimismo, en 1948 se dictó la Convención para la prevención y la sanción del delito de genocidio. En su Art. VI se dispuso la posibilidad de conformar una Corte penal internacional, sin perjuicio de la competencia que también pudiere corresponderle a los tribunales nacionales ${ }^{20}$.

de Asúa, Tratado, T. II, cit. nota n. 9, p. 1253 y ss. Allí expone su posición y la de otros autores. En general, Jiménez de Asúa se muestra bastante crítico con los juicios de Nuremberg, afirmando que el Derecho penal no puede atender comportamientos de esta naturaleza, mostrándose partidario de medidas bastante más radicales. Así se aprecia en Jiménez de Asúa, L., "Un comentario a la anunciada acción penal internacional”, en Revista de Ciencias Penales (Chile), X-XII 1944, p. 330 y ss. Para Jescheck, H.H./Weigend, T., Tratado de Derecho penal. Parte General. $5^{a}$ ed. (trad. M. Olmedo). Comares, Granada, 2002, p. 128; Jescheck, H.H., "El Tribunal Penal Internacional” (trad. Pifarré de Moner). Revista Penal, Nº 8, 2001, p. 54, el Tribunal no fue un verdadero órgano de jurisdicción internacional, pues sólo lo conformaron las fuerzas de ocupación, sin participación de Alemania, por lo que no tenía fuerza vinculante frente a este país; Cerezo Mir, J., Curso de Derecho penal español. Tecnos, T. I. $5^{\text {a }}$ ed. Madrid, 1996, p. 209-210. Asumiendo, en cambio, una postura muy favorable a los procesos de Nuremberg, Quintano Ripollés, Tratado, T. I, cit. nota n. 4, p. 417 y ss. Afirma en p. 427-428: "En lo tocante a lo procesal y prescindiendo de los reproches que puedan ser dirigidos al juicio de Nuremberg, en cuanto a su carácter de jurisdicción unilateral y ad hoc, a que varias veces se ha hecho referencia, es de destacar la suma escrupulosidad de la prueba, llevada a cabo con las máximas garantías de valoración y objetividad, según los mejores patrones del procedimiento criminal histórico".

${ }^{18}$ La Asamblea General de las Naciones Unidas por resolución número 177 (II) de 21 de noviembre de 1947 encomendó a la Comisión de Derecho Internacional que "formulase los principios del derecho internacional reconocidos en la Carta de los Juicios de Nuremberg y en las determinaciones del tribunal”. Ver Resolución número 177 (II) de 21 de noviembre de 1947 en http://daccessdds.un.org/doc/RESOLUTION/GEN/ NR0/041/15/IMG/NR004115.pdf?OpenElement (consultado el 5 de noviembre de 2009). Los principios elaborados por la Comisión de Derecho Internacional en 1950 en http://untreaty.un.org/ilc/texts/instruments/ english/draft\%20articles/7_1_1950.pdf (consultado el 5 de noviembre de 2009).

${ }^{19}$ El Zeidy, The principle, cit. nota n. 2, p. 83 y ss.; Stigen, The relationship, cit. nota n. 2, p. 31 y ss.

${ }^{20}$ Artículo VI: "Las personas acusadas de genocidio o de uno cualquiera de los actos enumerados en el artículo III, serán juzgadas por un tribunal competente del Estado en cuyo territorio el acto fue cometido, o ante la corte penal internacional que sea competente respecto a aquellas de las Partes contratantes que hayan reconocido su jurisdicción”. Al respecto, El Zeidy, M.M., "The principle of complementarity: a new machinery to implement international criminal law", en Michigan Journal of International Law, Vol. 23, 2001-2002, p. 876. 
Si bien se avanzó muy lentamente dado el contexto de Guerra Fría que imperaba, en 1974 la Asamblea General logró un consenso acerca del concepto de agresión ${ }^{21}$. Sin embargo, no fue sino hasta la década del noventa del siglo pasado, como consecuencia de la constitución de los tribunales ad hoc para la ex Yugoslavia y Rwanda, cuando recibieron un nuevo impulso los trabajos elaborados por la Comisión de Derecho Internacional. Es así que en 1994 sobre la base del proyecto de Estatuto de un Tribunal Penal Internacional aprobado por la Comisión, la Asamblea General de las Naciones Unidas procedió a designar una Comisión ad hoc. Ésta presentó un informe que sirvió de base para que la Asamblea General conformara un Comité preparatorio para el establecimiento de una Corte Penal Internacional (Preparatory Committee on the Establishment of an International Criminal Court ${ }^{22}$. Dicho Comité finalizó su trabajo con la sesión celebrada en marzoabril de 1998. Fue el texto aprobado en esta sesión el que sirvió de preparación para la Conferencia que habría de realizarse en Roma durante los meses de junio y julio de 1998, y que culminó, en definitiva, con el Estatuto para una Corte Penal Internacional ${ }^{23}$.

Centrando el examen en los Tribunales ad hoc, éstos fueron creados por resoluciones del Consejo de Seguridad de las Naciones Unidas. Tratándose de la antigua Yugoslavia por Resolución 827 de 25 de mayo de $1993^{24}$ y de Rwanda por Resolución 955 de 8 de noviembre de $1994^{25}$. Éstas se adoptaron de conformidad con el Capítulo VII de la Carta de las Naciones Unidas - "Acción en caso de amenazas a la paz, quebrantamientos de la paz o actos de agresión" ${ }^{26}$ - pues el Consejo de Seguridad consideró que dentro de sus facultades se comprendía el tomar medidas para el mantenimiento de la paz y, entre ellas, la de conformar Tribunales penales internacionales.

Pues bien, en lo que respecta a la determinación de la esfera competencial, los Estatutos de ambos tribunales ad hoc disponen de competencias concurrentes. En efecto, el art. 9 del Estatuto de Tribunal Penal Internacional para la ex Yugoslavia (TPIY) señala:

${ }^{21}$ Para un mayor detalle sobre este período, Carnevali, "Hacia la conformación”, cit. nota n. 7, p. 37-38; Mc Goldrick, D., "The permanent International Criminal Court: an end to the culture of impunity”, en Criminal Law Review, 1999, p. 628, p. 410-411; Gil Gil, A., Derecho penal internacional, Tecnos, Madrid, 1999, p. 60-61.

${ }^{22}$ Ambos, Justicia penal, cit. nota n. 6, p. 51 y ss. expone un cuadro comparativo de las disposiciones más importantes del Estatuto del Tribunal para la ex Yugoslavia, el proyecto de Estatuto de la Comisión y el proyecto alternativo.

${ }^{23}$ Cfr. Gil, Derecho penal, cit. nota n. 21, p. 61 y ss.; Ambos, Justicia penal, cit. nota n. 6, p. 17-20; El mismo. "Hacia el establecimiento de un Tribunal internacional permanente y un Código penal internacional. Observaciones desde un punto de vista del Derecho penal internacional”. (Trad. Gil/Bruzzone), en Actualidad penal, 1998-1, p. 225.

${ }^{24}$ Resolución 827 del Consejo de Seguridad de la Naciones Unidas http://daccessdds.un.org/doc/ UNDOC/GEN/N93/306/31/IMG/N9330631.pdf?OpenElement (consultado el 6 de noviembre de 2009).

25 Resolución 955 del Consejo de Seguridad de la Naciones Unidas http://daccessdds.un.org/doc/ UNDOC/GEN/N94/437/51/PDF/N9443751.pdf?OpenElement (consultado el 6 de noviembre de 2009).

${ }^{26}$ Capítulo VII de la Carta de las Naciones Unidas http://www.un.org/spanish/aboutun/charter/chapter7.htm (consultado el 6 de noviembre de 2009). 
“1. El Tribunal Internacional y los tribunales nacionales tendrán jurisdicción concurrente para enjuiciar a las personas que hayan cometido violaciones graves del derecho internacional humanitario en el territorio de la ex Yugoslavia a partir del 1 de enero de 1991.

2. El Tribunal Internacional tendrá primacía respecto de los Tribunales nacionales. En cualquier etapa del procedimiento, el Tribunal Internacional podrá pedir oficialmente a los Tribunales nacionales que convengan en la competencia del Tribunal Internacional de conformidad con el presente Estatuto y con las normas sobre procedimiento y prueba del Tribunal Internacional" 27.

Por su parte, el art. 8 del Estatuto del Tribunal Penal Internacional para Rwanda (TPIR), dispone lo que sigue:

“1. El Tribunal Internacional para Ruanda y las jurisdicciones nacionales son competentes simultáneamente para juzgar a los presuntos responsables de violaciones graves del derecho internacional humanitario cometidas en el territorio de Ruanda, así como a los ciudadanos ruandeses presuntamente responsables de tales violaciones cometidas en el territorio de Estados vecinos entre el $1^{\circ}$ de enero y el 21 de enero de 1994.

2. El Tribunal Internacional para Ruanda tiene prioridad sobre las jurisdicciones nacionales de todos los Estados. En cualquier momento del procedimiento puede solicitar oficialmente a las jurisdicciones nacionales que se desprendan de un procedimiento en su favor de acuerdo con el presente Estatuto y con su reglamento"28.

Consecuente con lo expuesto supra, ambos Estatutos reconocieron la competencia tanto de los tribunales ad hoc como de los tribunales nacionales. Empero, también se dispuso el principio de primacía de los internacionales, fundándose para ello en las razones esgrimidas para su instauración ${ }^{29}$. En efecto, considerando su especial propósito, a saber, contribuir con la restauración y mantenimiento de la paz, se requería de algo más que de competencias concurrentes ${ }^{30}$. Y es que en ese momento existían poderosos intereses humanitarios que imponían una jurisdicción extraordinaria, pues poco podía esperarse de los tribunales nacionales, dados los problemas para su funcionamiento; es decir, en ese momento histórico existían grandes probabilidades de que se impusiera

${ }^{27}$ Estatuto del TPIY: http://www.cruzroja.es/dih/pdf/Estatuto_Tribunal_Internacional_para_la_ex_ Yugoslavia.pdf (consultado el 11 de noviembre de 2009).

${ }^{28}$ Estatuto del TPIR http://www.derechos.org/nizkor/ley/ruanda.html (consultado el 11 de noviembre de 2009).

${ }^{29}$ El Zeidy, “The principle of complementarity”, cit. nota n. 20, p. 881 y ss.

${ }^{30}$ Sands, P., “After Pinochet: the role of national courts”, en Sands, P. (ed.). From Nuremberg to The Hague. The future of International Criminal Justice. University Press, Cambridge, 2003, p. 77-78; El Zeidy, The principle, cit. nota n. 2, p. 138; Brown, "Primacy or Complementarity", cit. nota n. 2, p. 394 y ss.; Cassese, International, cit. nota n. 1, p. 339. 
una cultura de la impunidad. También fue esa la razón para que los tribunales ad hoc se establecieran en ciudades lejanas al conflicto, pues facilitaba la imparcialidad y que los testigos pudieran declarar con mayor libertad ${ }^{31}$.

Otra de las razones que también se argumentaron a favor de la primacía de los tribunales ad hoc fue la de evitar una multiplicidad de tribunales ejerciendo su jurisdicción. Como se puso en evidencia con el ejemplo citado al inicio de este trabajo, son casos en los que pueden aplicarse diversos principios que justifican la competencia -así, el de territorialidad, personalidad pasiva, universalidad, por citar algunos-, por tanto, se hacía necesario disponer de una regla clara que determinara la competencia de uno de ellos por sobre los otros. En este orden de cosas, es evidente que una pluralidad de tribunales reclamando su competencia podía generar enormes dificultades en la recolección de pruebas, amén del riesgo de que pudieran ser destruidas ${ }^{32}$.

Es indudable que la primacía de los tribunales ad hoc daría lugar a particulares cuestionamientos, sobre todo por parte de aquellos que pretendían sustraerse de su competencia. Así ocurrió en el caso Tadic, donde la defensa planteó la improcedencia de la primacía por sobre los tribunales nacionales argumentando que se habría infringido la soberanía de los Estados, en cuanto son ellos los llamados a conocer los casos y que se violaba el principio jus de non evocando, por el cual el acusado sólo podía ser juzgado por tribunales ya establecidos y no por otros dispuestos posteriormente. Al respecto, debe tenerse presente que Tadic se encontraba arrestado en Alemania a la espera de ser juzgado por sus tribunales, y frente a la petición del TPIY invocando su primacía, lo entregó ${ }^{33}$. Pues bien, la Cámara de Apelaciones del TPIY resolvió, con fecha 2 de octubre de 1995, lo siguiente:

"Indeed, when an international tribunal such as the present one is created, it must be endowed with primacy over national courts. Otherwise, human nature being what it is, there would be a perennial danger of international crimes being characterised as "ordinary crimes" (Statute of the International Tribunal, art. 10, para. 2(a)), or proceedings being "designed to shield the accused", or cases not being diligently prosecuted (Statute of the International Tribunal, art. 10, para. 2(b)).

If not effectively countered by the principle of primacy, any one of those stratagems might be used to defeat the very purpose of the creation of an international criminal jurisdiction, to the benefit of the very people whom it has been designed to prosecute.

31 Tiefenbrun, "The paradox of International", cit. nota n. 7, p. 563 y ss.; Zahar, A.; Sluiter, G., International Criminal Court. University Press, Oxford, 2008, p. 450-454.

32 Brown, "Primacy or Complementarity", cit. nota n. 2, p. 398.

33 Sassoli, M.; Olson, L.M., "Prosecutor v. Tadic", en American Journal of International Law, Vol. 94, 2000, p. 571 y ss.; Murphy, Sean D. "Progress and Jurisprudence of the International Criminal Tribunal for the former Yugoslavia", en American Journal of International Law, Vol. 93, 1999, p. 58-59; Brown, "Primacy or Complementarity”, cit. nota n. 2, p. 403 y ss.; Sands, “After Pinochet”, cit. nota n. 30, p. 78-79. 
The principle of primacy of this International Tribunal over national courts must be affirmed; the more so since it is confined within the strict limits of Articles 9 and 10 of the Statute and Rules 9 and 10 of the Rules of Procedure of the International Tribunal" 34 .

Tal como se expuso precedentemente, resulta claro que la primacía se dirige a dotar al órgano internacional de los necesarios instrumentos que le permitan actuar con eficacia, pues de otro modo se vería imposibilitado de juzgar los delitos internacionales. El imputado podría sustraerse fácilmente de la persecución.

En cuanto a los cuestionamientos al principio jus de non evocando, el mismo fallo señala:

"63. The objection founded on the theory of jus de non evocando was considered by the Trial Chamber which disposed of it in the following terms: "Reference was also made to the jus de non evocando, a feature of a number of national constitutions. But that principle, if it requires that an accused be tried by the regularly established courts and not by some special tribunal set up for that particular purpose, has no application when what is in issue is the exercise by the Security Council, acting under Chapter VII, of the powers conferred upon it by the Charter of the United Nations. Of course, this involves some surrender of sovereignty by the member nations of the United Nations but that is precisely what was achieved by the adoption of the Charter". (Decision at Trial, at para. 37.)"35.

Haciendo suyo lo resuelto por la Cámara de Primera Instancia, la Cámara de Apelaciones resaltó que no se trataba de cualquier tribunal, sino de uno que surge de una resolución del Consejo de Seguridad de las Naciones Unidas. Por tanto, ya los Estados, en un ejercicio soberano, habían dotado al organismo nacional de tales facultades.

En el TPIR también se plantearon problemas similares ${ }^{36}$. Es así que 1996 Elizaphan Ntakirutimana, un hutu de Rwanda, fue acusado de la matanza de cerca de 10.000 tutsis. Como se encontraba viviendo en Texas, Estados Unidos, el TPIR requirió a dicho país su entrega. Es del caso que un juez federal consideró que existían pruebas suficientes que fundamentaban los cargos y que los hechos también eran delitos bajo la ley norteamericana, por lo que determinó su arresto. Por su parte, el gobierno de Clinton se manifestaba muy partidario de colaborar con el TPIR, reconociendo con ello su primacía. Sin embargo, el defensor de Ntakirutimana -incluso llegó a ser defendido por el ex fiscal general Ramsey Clark-argumentó que el tribunal internacional, creado por una resolución de las

${ }^{34}$ Decision on the defence motion for interlocutory appeal on jurisdiction (caso Tadic IT-94-1) http:// www.icty.org/x/cases/tadic/acdec/en/51002.htm (consultado el 11 de noviembre de 2009).

${ }^{35}$ Decision on the defence motion for interlocutory appeal on jurisdiction (caso Tadic IT-94-1) http:// www.icty.org/x/cases/tadic/acdec/en/51002.htm (consultado el 11 de noviembre de 2009).

36 Payam, A., "The international Criminal Tribunal for Rwanda: the Politics and Pragmatic of Punishment”, en American Journal of International Law, Vol. 90, 1996, p. 510 y ss. 
Naciones Unidas, vulneraba garantías constitucionales. Tales consideraciones encontraron apoyo en un juez de Texas. Dicho juez determinó que era inconstitucional cooperar con el TPIR y por tanto improcedente la entrega de Ntakirutimana al TPIR, ya que no existía un tratado de extradición entre los Estados Unidos y el TPIR ${ }^{37}$. Asimismo, no se habrían probado los cargos criminales atribuidos ${ }^{38}$.

Finalmente, la Suprema Corte de los Estados Unidos determinó la procedencia de la entrega al TPIR, desestimando los argumentos recién expuestos, sobre la base de que ya existían disposiciones en la legislación norteamericana que permitían la entrega. Cabe señalar que fue la primera persona que Estados Unidos entregó a un tribunal internacional para su juzgamiento ${ }^{39}$.

Para comprender adecuadamente el sentido y alcance del principio de primacía, que fundamenta la actuación de los tribunales ad hoc, deben tenerse en cuenta las reglas de procedimiento y de evidencia ${ }^{40}$. Precisamente, en dichos textos -los que han sido modificados en diversas oportunidades- se disponen los procedimientos para resolver cuándo y de qué forma un caso puede ser conocido por los tribunales internacionales. Es así que tratándose del TPIY la regla 9 dispone qué circunstancias son requeridas para que un caso pueda ser remitido al tribunal internacional aun cuando ya esté siendo conocido por uno nacional. Entre las razones que se pueden invocar está el hecho de que el delito sea calificado como común - "ordinary crime"-, o se observe falta de imparcialidad o independencia.

Si bien tales disposiciones autorizaban la actuación del TPIY, comenzó a apreciarse que no se disponía de mecanismos claros para la selección de casos y que de alguna forma quedaba entregado a la discrecional del órgano persecutor ${ }^{41}$. Por otro lado, si bien en un inicio se apreciaba un legítimo interés de abarcar gran parte de los casos, sobre todo considerando que los países en conflicto no ofrecían garantías de que podían impartir juicios justos -se apreciaba una fuerte desconfianza hacia los tribunales internos- ${ }^{42}$, se comenzó a valorar que no podía pretenderse abarcarlo todo. En consecuencia, no era posible prescindir del apoyo que podía provenir de los tribunales nacionales. Sin renunciar

${ }^{37}$ Sobre las obligaciones de los Estados Unidos, Kushen, R.; Harris, K.J., "Surrender of fugitives by the United status to the war crimes Tribunals for Yugoslavia and Rwanda", en American Journal of International Law, Vol. 90, 1996, p. 501 y ss.

${ }^{38}$ Coombs, M., "In re surrender of Ntakirutimana”, en American Journal of International Law, Vol. 94, 2000, p. 171 y ss.

Brown, "Primacy or Complementarity", cit. nota n. 2, p. 411 y ss.

${ }^{39}$ Prosecutor V. Elizaphan And Gérard Ntakirutimana, Cases No. ICTR-96-10 \& ICTR-96-17-T http:// www.ictr.org/default.htm (consultado el 12 de noviembre de 2009).

40 Respecto del TPIY http://www.icty.org/x/file/Legal\%20Library/Rules_procedure_evidence/ IT032_Rev43_en.pdf (consultado el 12 de noviembre de 2009). Para el TPIR http://www.ictr.org/ENGLISH/ rules/080314/080314.pdf (consultado el 12 de noviembre de 2009).

41 Así, El Zeidy, The principle, cit. nota n. 2, p. 143, quien destaca que una de las grandes diferencias entre los tribunales ad hoc y la Corte Penal Internacional está en el sistema de admisibilidad. Este último dispone de filtros que los otros carecen.

${ }^{42}$ Kleffner, Complementarity, cit. nota n. 13, p. 66. 
a la primacía debía disponerse de reglas que permitieran un mayor protagonismo de los órganos jurisdiccionales nacionales.

Justamente y en esta misma dirección, en la sesión del Consejo de Seguridad celebrada el 23 de julio de 2002 el Presidente del Consejo formuló la siguiente declaración en nombre del Consejo:

“(...)El Consejo de Seguridad hace suya, por consiguiente, la estrategia general presentada en el informe para trasladar a las jurisdicciones nacionales competentes las causas relativas a acusados de rango intermedio e inferior como la práctica que probablemente sea la mejor forma de permitir al Tribunal Penal Internacional para la ex Yugoslavia alcanzar su objetivo actual de terminar todas las actividades judiciales en primera instancia para 2008 " 43.

El propósito evidente era que el TPIY se concentrara en los casos más relevantes, respecto de los mayores responsables por crímenes internacionales, siguiendo así un camino similar al recorrido por el Tribunal Militar Internacional de Nuremberg ${ }^{44}$. La estrategia expuesta se vio refrendada por dos resoluciones del Consejo de Seguridad, 1503 de $2003^{45}$ y 1534 de $2004^{46}$. No obstante, el cambio más notable tuvo lugar con las modificaciones a la regla 11 bis relativa al procedimiento y la evidencia.

Tratándose de las resoluciones del Consejo de Seguridad se señaló:

"Insta a cada uno de los Tribunales a que, al examinar y confirmar las acusaciones nuevas, se aseguren de que concentran la labor en el procesamiento de los más altos dirigentes de quienes se sospeche que les cabe la mayor responsabilidad respecto de los delitos que sean de competencia del Tribunal de que se trate".

En lo que respecta a la regla 11 bis $^{47}$, se disponen determinados criterios por los cuales pueden actuar los tribunales nacionales, permitiendo a los tribunales ad hoc intervenir si se aprecia que no se están cumpliendo con los objetivos trazados. Es así, por

${ }^{43}$ Sesión del Consejo de Seguridad de las Naciones Unidas de 23 de julio de 2002. http://daccessdds-ny.un.org/doc/UNDOC/GEN/N02/491/50/PDF/N0249150.pdf?OpenElement (consultado el 13 de noviembre de 2009).

${ }^{44}$ Meron, "Reflections", cit. nota n. 8, p. 563; El Zeidy, The principle, cit. nota n. 2, p. 146.

${ }^{45}$ Sesión del Consejo de Seguridad de 28 de agosto de 2003 http://www.unhchr.ch/Huridocda/Huridoca. nsf/0/282ee21ff552a976c1256d9400293811?Opendocument (consultado el 13 de noviembre de 2009).

46 Sesión del Consejo de Seguridad de 26 de marzo de 2004 http://www.unhcr.org/cgi-bin/texis/vtx/ refworld/rwmain/opendocpdf.pdf?reldoc=y\&docid=4a2935f92 (consultado el 13 de noviembre de 2009).

47 Referral of the Indictment to Another Court

(A) After an indictment has been confirmed and prior to the commencement of trial, irrespective of whether or not the accused is in the custody of the Tribunal, the President may appoint a bench of three Permanent Judges selected from the Trial Chambers (hereinafter referred to as the "Referral Bench"), which solely and exclusively shall determine whether the case should be referred to the authorities of a State:

(i) in whose territory the crime was committed; or

(ii) in which the accused was arrested; or 
ejemplo, que en el caso Jankovic se señaló que las reglas 11 bis D (iv) y 11 bis F establecen medidas respecto de insuficiencias en la persecución o atentados al debido proceso que puedan apreciarse en los casos que se sigan en los tribunales nacionales ${ }^{48}$. En términos similares se pronunció el TPIY en el caso Stankovic ${ }^{49}$. Ambos fueron juzgados por tribunales de Bosnia y Herzegovina al apreciarse que podían garantizar una persecución diligente y un debido proceso ${ }^{50}$.

De lo expuesto, puede decirse que hoy los tribunales ad hoc han adoptado un modelo similar - no idéntico- al consagrado en el Estatuto de Roma, esto es, la complementariedad. Es decir, si se resuelve que los tribunales internos no han sido diligentes en la

(iii) having jurisdiction and being willing and adequately prepared to accept such a case, so that those authorities should forthwith refer the case to the appropriate court for trial within that State.

(B) The Referral Bench may order such referral proprio motu or at the request of the Prosecutor, after having given to the Prosecutor and, where applicable, the accused, the opportunity to be heard and after being satisfied that the accused will receive a fair trial and that the death penalty will not be imposed or carried out.

(C) In determining whether to refer the case in accordance with paragraph (A), the Referral Bench shall, in accordance with Security Council resolution 1534 (2004), consider the gravity of the crimes charged and the level of responsibility of the accused.

(D) Where an order is issued pursuant to this Rule:

(i) the accused, if in the custody of the Tribunal, shall be handed over to the authorities of the State concerned;

(ii) the Referral Bench may order that protective measures for certain witnesses or victims remain in force;

(iii) the Prosecutor shall provide to the authorities of the State concerned all of the information relating to the case which the Prosecutor considers appropriate and, in particular, the material supporting the indictment; behalf.

(iv) the Prosecutor may send observers to monitor the proceedings in the national courts on her

(E) The Referral Bench may issue a warrant for the arrest of the accused, which shall specify the State to which he is to be transferred to trial.

(F) At any time after an order has been issued pursuant to this Rule and before the accused is found guilty or acquitted by a national court, the Referral Bench may, at the request of the Prosecutor and upon having given to the State authorities concerned the opportunity to be heard, revoke the order and make a formal request for deferral within the terms of Rule 10 .

(G) Where an order issued pursuant to this Rule is revoked by the Referral Bench, it may make a formal request to the State concerned to transfer the accused to the seat of the Tribunal and the State shall accede to such a request without delay in keeping with Article 29 of the Statute. The Referral Bench or a Judge may also issue a warrant for the arrest of the accused.

(H) A Referral Bench shall have the powers of, and insofar as applicable shall follow the procedures laid down for, a Trial Chamber under the Rules

(I) An appeal by the accused or the Prosecutor shall lie as of right from a decision of the Referral Bench whether or not to refer a case. Notice of appeal shall be filed within fifteen days of the decision unless the accused was not present or represented when the decision was pronounced, in which case the time-limit shall run from the date on which the accused is notified of the decision.

${ }^{48}$ Decision on referral of case under rule 11 bis (caso Jankovic IT-96-23/2) de 15 de noviembre de 2005 http://www.icty.org/x/cases/stankovic/acdec/en/051115.htm (consultado el 13 de noviembre de 2009).

${ }^{49}$ Decision on referral of case under rule 11 bis (caso Stankovic IT-96-23/2) de 1 de septiembre de 2005 http://www.icty.org/x/cases/stankovic/acdec/en/050901.htm (consultado el 13 de noviembre de 2009).

${ }^{50} \mathrm{Al}$ respecto, Kleffner, Complementarity, cit. nota n. 13, p. 69. 
persecución de los delitos, éstos pueden ser conocidos por los tribunales internacionales ${ }^{51}$. La excepción se presenta cuando se trata de los grandes responsables, puesto que éstos deben ser juzgados por el tribunal internacional. Así aconteció con Radovan Karadzic y se espera que suceda con Ratko Mladic cuando sea aprehendido. Lo expuesto pone de manifiesto que no es posible trazar nociones de primacía y complementariedad exclusivas y excluyentes. Por el contrario, caminan una al lado de la otra. Es más, la ya citada regla 11 bis aunque dispone un sistema de complementariedad lo hace dentro de un contexto en que el TPIY mantiene su primacía 52 .

Así puede desprenderse de lo resuelto por la Cámara de Primera Instancia del TPIR en el caso Ntuyahaga:

“(...) although it accepts the submissions of the Prosecutor and the Belgian Government inasmuch as the Tribunal does not have exclusive jurisdiction over crimes included in its mandate and that its criminal proceedings are complementary to those of national jurisdictions, it wishes to underscore that, in its opinion, and as submitted by the Defence, the principle of concurrent jurisdiction as provided in paragraph (1) of Article 8 of the Statute, which recognizes the complementary nature of the judicial work performed by the Tribunal and national courts, must be read together with the provisions of paragraph 2 of said Article 8, which confers upon the Tribunal primacy over the national courts of all States;" 53 .

\section{El PRinCiPio de COMPlementariedad EN El Estatuto de Roma}

La conformación de los tribunales ad hoc y las discusiones hacia la instauración de una Corte Penal Internacional (CPI) han marcado el fin de las prerrogativas fundadas en la soberanía nacional, con todo su marco de discrecionalidad y arbitrariedad que supone ${ }^{54}$; esto es, existen ciertos delitos en que la comunidad internacional manifiesta su interés en que deben ser juzgados, ya sea por tribunales nacionales o internacionales. La cuestión que debe resolverse no es pues si pueden ser objeto de investigación, sino cómo y por quiénes. Por tanto, crucial resulta precisar los mecanismos de determinación de las competencias entre los tribunales nacionales y los internacionales. Precedentemente se ha analizado cuáles han sido los pasos seguidos por tribunales que se han instaurado ex post, a saber, los de Nuremberg, el TPIY y el TPIR. Un camino diverso dispuso

${ }^{51}$ El Zeidy, The principle, cit. nota n. 2, p. 149, destaca que la jurisprudencia de los tribunales ad hoc ha sido poco clara en la definición de la "persecución diligente".

52 En estos términos, El Zeidy, The principle, cit. nota n. 2, p. 151.

53 Decision on the prosecutor's motion to withdraw the indictment (caso Ntuyahaga ICTR-98-40-T) de 18 de marzo de 1999. http://www.ictr.org/default.htm (consultado el 13 de noviembre de 2009). Citado también por El Zeidy, The principle, cit. nota n. 2, p. 151.

${ }^{54}$ Brown, "Primacy or Complementarity", cit. nota n. 2, p. 430. 
el Estatuto de Roma, por el que se implementa la CPI, al consagrar el principio de complementariedad.

Respecto de la Corte Especial para Sierra Leona ${ }^{55}$ y el Tribunal Especial para El Líbano $^{56}$ se consagra su primacía, pero sólo sobre sus propios tribunales.

Si bien en 1994, durante las discusiones de la Comisión ad hoc para el establecimiento de la Corte Penal Internacional, algunos Estados se manifestaron favorables a la primacía de la CPI, de manera similar a los recién creados tribunales internacionales para la ex Yugoslavia y Rwanda, no tuvieron mayor acogida ${ }^{57}$. En efecto, se tenía presente que los tribunales ad hoc fueron implementados por especiales circunstancias -como ya se señaló- y, además, no podían dejarse de lado las reticencias que podía generar un tribunal permanente por sobre los Estados. Es por ello que los trabajos emprendidos por el Comité preparatorio para el establecimiento de una Corte Penal Internacional (Preparatory Committee on the Establishment of an International Criminal Court) en 1996 se iniciaron con cierto consenso sobre este punto: la complementariedad entre la CPI y los tribunales internos ${ }^{58}$. Es más, se tuvo cierto cuidado en dejar claro que las prerrogativas jurisdiccionales eran de los Estados y que la CPI sólo debía actuar excepcionalmente ${ }^{59}$. Por tanto, los trabajos se dirigieron a establecer cuáles serían los mecanismos para autorizar la competencia de la CPI. En este orden, en agosto de 1997 el Comité preparatorio trabajó en un proyecto de artículo -Art. 35-, en donde se precisaban las cuestiones de admisibilidad $^{60}$. Pues bien, allí ya se individualizaban ciertos criterios que luego se materializaron en el Estatuto de Roma, a saber, cuando el Estado no puede o no desea ejercer su jurisdicción -the unwillingness or inability of the State genuinely to prosecute- ${ }^{61}$.

Estimo que el iter seguido por la Comité preparatorio y luego por la Conferencia de Roma fue el correcto. Es muy probable que de haberse planteado la idea de una primacía

55 Creado por Resolución 1315 de 14 de agosto de 2000 del Consejo de Seguridad de las Naciones Unidas http:/www.unhcr.org/cgi-bin/texis/vtx/refworld/rwmain/opendocpdf.pdf? reldoc $=$ y\&docid= $4844 \mathrm{fb} 432$ (consultado el 13 de noviembre de 2009).

56 Creado por Resolución 1757 de 30 de mayo de 2007 del Consejo de Seguridad de las Naciones Unidas http:/www.unhcr.org/cgi-bin/texis/vtx/refworld/rwmain/opendocpdf.pdf?reldoc=y\&docid=47e0d26d2 (consultado el 13 de noviembre de 2009).

${ }^{57}$ Stigen, The relationship, cit. nota n. 2, p. 64; Fernández, S.A. "Elementos para el establecimiento de una Corte Penal Internacional eficaz e independiente", en Revista IIDH (Instituto Interamericano de Derechos Humanos), $\mathrm{N}^{\circ} 23,1996$, p. 52-53.

58 Stigen, The relationship, cit. nota n. 2, p. 69; Brown, "Primacy or Complementarity", cit. nota n. 2 , p. 423.

59 Basta apreciar los primeros trabajos realizados por el Comité preparatorio en 1996 http://www. iccnow.org/? mod=prepcommittee (consultado el 18 de noviembre de 2009). Incluso, ONG como Human Rights Watch http://www.iccnow.org/documents/1PrepCmtCommentaryHRW.pdf (consultado el 18 de noviembre de 2009) e International Commission of Jurists http://www.iccnow.org/documents/1PrepCmt 3rdPositionPaperICJ.pdf (consultado el 18 de noviembre de 2009), se mostraron partidarios de configurar un sistema de complementariedad.

${ }^{60}$ Artículo 35 del proyecto elaborado por el Comité preparatorio http://www.iccnow.org/documents/ IssuesofAdmissibility.pdf (consultado el 18 de noviembre de 2009).

${ }^{61}$ Con detalle, Stigen, The relationship, cit. nota n. 2, p. 74 y ss.; El Zeidy, The principle, cit. nota n. 2, p. 126 y ss.; 
de la CPI por sobre los tribunales nacionales habría derivado en un fracaso absoluto. Pueden apreciarse argumentos de orden práctico y de conveniencia política. En efecto, razones de carácter práctico obligan a contar con la actuación de los Estados nacionales. $\mathrm{Y}$ es que la CPI no puede hacerse cargo de todos los casos en que se cometan crímenes de derecho internacional. Consideraciones que, como se expuso supra, también fueron valoradas en su momento respecto de los tribunales ad hoc.

En esta línea, no puede prescindirse de la importancia que en este sentido tiene el principio de territorialidad, no sólo por la mayor eficacia preventivo general, sino y por sobre todo la mejor capacidad de que pueden disponer las instituciones nacionales para una más acuciosa investigación y eventual juzgamiento ${ }^{62}$.

Por otro lado, razones de orden político llaman a mantener la actuación principal del Estado. Los Estados son particularmente celosos en el resguardo de su soberanía punitiva. Es por ello que la CPI no pretende socavar o disminuir la competencia que les corresponde a los Estados, pudiendo éstos emplear todas las herramientas, "así, extradición, u otro tipo de cooperación entre Estados" que sean necesarias para la persecución y juzgamientos de los delitos ${ }^{63}$. Probablemente, tales cuestionamientos se habrían presentado de haberse adoptado otro modelo de complementariedad, en donde la competencia de la CPI se determinaba sobre la base de un determinado grupo de delitos ${ }^{64}$.

\section{Conclusiones finales}

Durante la Segunda Guerra Mundial, a través de la Declaración de Moscú, uno de los mayores problemas que se presentaron fue el precisar cómo serían juzgados los crímenes cometidos por las potencias del Eje. Si quedaban entregados a los tribunales nacionales, sobre la base de la territorialidad, o si se conformaba un tribunal internacional. Luego el Estatuto de Londres dispuso que atendiendo a ratione loci-crímenes no tuvieren localización geográfica particular- y a ratione personae -criminales principalesdebía ser un Tribunal Militar Internacional el llamado a su juzgamiento, dejando que los otros casos pudieran ser atendidos por tribunales internos. Se trataba pues de una especie de complementariedad, en que el Tribunal Militar Internacional se reservaba los casos más importantes.

Pocos años después, la Convención para la prevención y la sanción del delito de genocidio de 1948 también reguló un sistema propio de complementariedad, esto es, la competencia es de los tribunales nacionales, atendiendo la territorialidad, sin perjuicio de que también pudiera ser conocido por una Corte Penal Internacional. Sin embargo,

${ }^{62}$ Brown, "Primacy or Complementarity", cit. nota n. 2, p. 391-392.

${ }^{63}$ Al respecto, Carnevali R., "El principio de subsidiariedad y su papel en la determinación de las competencias sancionatorias de la Unión Europea. Relación con el principio de complementariedad de la Corte Penal Internacional”, en Zeitschrift für Internationale Strafrechtsdogmatik 6/2009 (www.zis-online.com), p. 283-284 (consultado el 18 de noviembre de 2009).

${ }^{64}$ Brown, "Primacy or Complementarity", cit. nota n. 2, p. 390. 
no se abordó mayormente, por lo que los principales actores llamados a conocer de estos casos debían ser los Estados nacionales.

Como criterio opuesto al de complementariedad fue el que se dispuso respecto de los tribunales ad hoc durante la década de los noventa. En efecto, el propósito perseguido con su instauración fue el de que ejercieran una estricta primacía. Sin embargo, con el paso del tiempo fueron derivando hacia una suerte de complementariedad con los tribunales nacionales - puede que haya sido una decisión inadvertida- ${ }^{65}$, al apreciar las enormes dificultades que representaba conocer todos los casos. En este sentido, fundamentales resultaron las resoluciones del Consejo de Seguridad 1503 y 1534, y particularmente la regla 11 bis. De algún modo, se dispuso de un sistema similar al seguido por el Tribunal Militar Internacional y lo resuelto por el Consejo de Control Aliado a través de la Ley $\mathrm{N}^{\circ} 10$ de 20 de diciembre de 1945.

La experiencia vivida por los tribunales ad hoc hicieron ver que la CPI no podía transitar un camino similar, esto es, haber pretendido dotarla de primacía sobre los tribunales internos. Es por ello que se dispone de un sistema de complementariedad: la CPI sólo tiene un carácter complementario respecto de las jurisdicciones nacionales. Son los Estados los que deben juzgar a los responsables por crímenes internacionales y sólo cuando no puedan o no desean hacerlo, le corresponderá intervenir a la CPI -art. 17 del Estatuto-.

Por lo expuesto, es preciso resaltar que las competencias de los Estados no se ven en absoluto cuestionadas, por el contrario, se complementan con las de la CPI - no se produce una cesión de competencias-. En caso de actuación de la CPI cuenta a su vez con la cooperación de los Estados, conforme se dispone de las obligaciones contraídas al haber ratificado el Estatuto de Roma.

\section{BIBLIOGRAFÍA}

\section{Libros}

Ambos, K., La nueva justicia penal internacional. Fundación Myrna Mack, Guatemala, 2000.

Cassese, A., International Criminal Law, University Press, $2^{\mathrm{a}}$ ed. Oxford, 2008.

Cerezo Mir, J., Curso de Derecho penal español. Tecnos, T. I. $5^{\text {a }}$ ed. Madrid, 1996.

EL ZEIDy, M.M., The principle of Complementarity in International Criminal Law, Nijhoff Publishers, Leiden-Boston, 2008.

Gil GiL, A., Derecho penal internacional, Madrid: Tecnos, 1999.

Jescheck, H.H.; Weigend, T., Tratado de Derecho penal. Parte General. $5^{\text {a }}$ ed. (trad. M. Olmedo). Comares, Granada, 2002.

Jiménez de AsúA, L., Tratado de Derecho penal. T. II. $5^{a}$ ed. Losada, Buenos Aires, 1992.

KLEFFner, J.K., Complementarity in the Rome Statute and National Criminal Jurisdictions. University Press, Oxford, 2008.

${ }^{65}$ El Zeidy, The principle, cit. nota n. 2, p. 153. 
Matus, J.P., "La política criminal de los tratados internacionales", en Carnevali, R., (Coord.). Cuestiones de Política Criminal en los tiempos actuales. Editorial Jurídica, Santiago, 2009.

Quintano Ripollés, A., Tratado de Derecho penal internacional e internacional penal. T. I. Madrid, Madrid, 1955.

SANDs, P., "After Pinochet: the role of national courts", en Sands, P. (ed.). From Nuremberg to The Hague. The future of International Criminal Justice. Cambridge: University Press, 2003.

STIGEN, J., The relationship between the International Criminal Court and National Jurisdictions. The Principle of Complementarity, Nijhoff Publishers, Leiden-Boston, 2008.

Zahar, A.; Sluiter, G., International Criminal Court. University. Press, Oxford, 2008.

\section{Revistas}

Амвos, K., "Hacia el establecimiento de un Tribunal internacional permanente y un Código penal internacional. Observaciones desde un punto de vista del Derecho penal internacional". (Trad. Gil/Bruzzone), en Actualidad penal, 1998-1.

Brown, B. "Primacy or Complementarity: Reconciling the Jurisdiction of National Courts and International Criminal Tribunals", en The Yale Journal of International Law, Vol. 23, 1998.

Carnevali, R., "El principio de subsidiariedad y su papel en la determinación de las competencias sancionatorias de la Unión Europea. Relación con el principio de complementariedad de la Corte Penal Internacional", en Zeitschrift für Internationale Strafrechtsdogmatik 6/2009 (www.zis-online.com), p. 283-284 (consultado el 18 de noviembre de 2009).

Carnevali, R., "Hacia la conformación de un Tribunal penal internacional. Evolución histórica y desafíos futuros", en Revista de Derecho de la Universidad Católica del Norte. Sede Coquimbo. $\mathrm{N}^{\circ} 10,2003$.

Cassese, A., "Opinion: The International Criminal Tribunal for the Former Yugoslavia and Human Rights", en European Human Rights Law Review, 1997.

Coombs, M., "In re surrender of Ntakirutimana", en American Journal of International Law, Vol. 94, 2000.

Donnedieu, H., "Le procès de Nuremberg", en Revue de Science Criminelle et de Droit Pénal Comparé, $\mathrm{N}^{\circ}$ 2, 1947.

EL ZEIDY, M.M., "The principle of complementarity: a new machinery to implement international criminal law", en Michigan Journal of International Law, Vol. 23, 2001-2002.

FERnÁndez, S.A., "Elementos para el establecimiento de una Corte Penal Internacional eficaz e independiente", en Revista IIDH (Instituto Interamericano de Derechos Humanos), No 23, 1996.

GlueCK, S., "By what Tribunal shall war offenders be tried?", en Harvard Law Review. Vol. 56, 1942-1943.

JimÉnEZ DE AsúA, L., "Un comentario a la anunciada acción penal internacional”, en Revista de Ciencias Penales (Chile), X-XII 1944.

Jescheck, H.H., "El Tribunal Penal Internacional" (trad. Pifarré de Moner). Revista Penal, $\mathrm{N}^{\circ} 8,2001$.

Kushen, R.; Harris, K.J., "Surrender of fugitives by the United status to the war crimes Tribunals for Yugoslavia and Rwanda”, en American Journal of International Law, Vol. 90, 1996.

MC Goldrick, D., "The permanent International Criminal Court: an end to the culture of impunity", en Criminal Law Review, 1999, p. 628.

Meron, T., "Reflections on the prosecution of war crimes by International Tribunals", en American Journal of International Law, Vol. 100, 2006. 
Murphy, SEAn D., "Progress and Jurisprudence of the International Criminal Tribunal for the former Yugoslavia", en American Journal of International Law, Vol. 93, 1999.

Payam, A., "The international Criminal Tribunal for Rwanda: the Politics and Pragmatic of Punishment", en American Journal of International Law, Vol. 90, 1996.

Sassoli, M.; Olson, L.M., "Prosecutor v. Tadic", en American Journal of International Law, Vol. 94, 2000.

Tiefenbrun, S.W., "The paradox of International adjudication: developments in the International Criminal Court for the Former Yugoslavia and Rwanda, the World Court and the International Criminal Court", en North Carolina Journal of International Law and commercial regulation, No 3, 2000.

\section{Páginas web}

La Declaración de Moscú en http://avalon.law.yale.edu/wwii/moscow.asp (consultado el 5 de noviembre de 2009).

Estatuto de Londres en http://avalon.law.yale.edu/imt/imtchart.asp (consultado el 5 de noviembre de 2009).

El texto de constitución del Tribunal Militar Internacional en http://avalon.law.yale.edu/imt/ imtconst.asp (consultado el 5 de noviembre de 2009).

Ley $\mathrm{N}^{\circ} 10$ de 20 de diciembre de 1945 en http://avalon.law.yale.edu/imt/imt10.asp (consultado el 5 de noviembre de 2009).

American Journal of International Law, Vol. 41, 1947, p. 172 y ss. También en http://avalon.law. yale.edu/subject_menus/judcont.asp (consultado el 5 de noviembre de 2009).

La Asamblea General de las Naciones Unidas. Resolución número 177 (II) de 21 de noviembre de 1947 en http://daccessdds.un.org/doc/RESOLUTION/GEN/NR0/041/15/IMG/NR004115. pdf?OpenElement (consultado el 5 de noviembre de 2009).

Los principios elaborados por la Comisión de Derecho Internacional en 1950 en http://untreaty. un.org/ilc/texts/instruments/english/draft\%20articles/7_1_1950.pdf (consultado el 5 de noviembre de 2009).

Resolución 827 del Consejo de Seguridad de la Naciones Unidas http://daccessdds.un.org/doc/ UNDOC/GEN/N93/306/31/IMG/N9330631.pdf?OpenElement (consultado el 6 de noviembre de 2009).

Resolución 955 del Consejo de Seguridad de la Naciones Unidas http://daccessdds.un.org/doc/ UNDOC/GEN/N94/437/51/PDF/N9443751.pdf?OpenElement (consultado el 6 de noviembre de 2009).

Capítulo VII de la Carta de las Naciones Unidas http://www.un.org/spanish/aboutun/charter/ chapter7.htm (consultado el 6 de noviembre de 2009).

Estatuto del TPIY: http://www.cruzroja.es/dih/pdf/Estatuto_Tribunal_Internacional_para_la_ ex_Yugoslavia.pdf (consultado el 11 de noviembre de 2009).

Estatuto del TPIR http://www.derechos.org/nizkor/ley/ruanda.html (consultado el 11 de noviembre de 2009).

Decision on the defence motion for interlocutory appeal on jurisdiction (caso Tadic IT-94-1) http://www.icty.org/x/cases/tadic/acdec/en/51002.htm (consultado el 11 de noviembre de 2009).

Decision on the defence motion for interlocutory appeal on jurisdiction (caso Tadic IT-94-1) http://www.icty.org/x/cases/tadic/acdec/en/51002.htm (consultado el 11 de noviembre de 2009). 
Prosecutor V. Elizaphan And Gérard Ntakirutimana, Cases No. ICTR-96-10 \& ICTR-96-17-T http://www.ictr.org/default.htm (consultado el 12 de noviembre de 2009).

Respecto del TPIY http://www.icty.org/x/file/Legal\%20Library/Rules_procedure_evidence/ IT032_Rev43_en.pdf (consultado el 12 de noviembre de 2009).

Para el TPIR http://www.ictr.org/ENGLISH/rules/080314/080314.pdf (consultado el 12 de noviembre de 2009).

Sesión del Consejo de Seguridad de las Naciones Unidas de 23 de julio de 2002. http://daccess-ddsny.un.org/doc/UNDOC/GEN/N02/491/50/PDF/N0249150.pdf?OpenElement (consultado el 13 de noviembre de 2009).

Sesión del Consejo de Seguridad de 28 de agosto de 2003 http://www.unhchr.ch/Huridocda/ Huridoca.nsf/0/282ee21ff552a976c1256d9400293811?Opendocument (consultado el 13 de noviembre de 2009).

Sesión del Consejo de Seguridad de 26 de marzo de 2004 http://www.unhcr.org/cgi-bin/texis/ $\mathrm{vtx} / \mathrm{refworld} / \mathrm{rwmain} /$ opendocpdf.pdf? reldoc=y\&docid=4a2935f92 (consultado el 13 de noviembre de 2009).

Decision on referral of case under rule 11 bis (caso Jankovic IT-96-23/2) de 15 de noviembre de $2005 \mathrm{http} / /$ www.icty.org/x/cases/stankovic/acdec/en/051115.htm (consultado el 13 de noviembre de 2009).

Decision on referral of case under rule 11 bis (caso Stankovic IT-96-23/2) de 1 de septiembre de 2005 http://www.icty.org/x/cases/stankovic/acdec/en/050901.htm (consultado el 13 de noviembre de 2009).

Decision on the prosecutor's motion to withdraw the indictment (caso Ntuyahaga ICTR-98-40-T) de 18 de marzo de 1999. http://www.ictr.org/default.htm (consultado el 13 de noviembre de 2009).

Resolución 1315 de 14 de agosto de 2000 del Consejo de Seguridad de las Naciones Unidas http:// www.unhcr.org/cgi-bin/texis/vtx/refworld/rwmain/opendocpdf.pdf? reldoc =y\&docid=4844fb432 (consultado el 13 de noviembre de 2009).

Resolución 1757 de 30 de mayo de 2007 del Consejo de Seguridad de las Naciones Unidas http:// www.unhcr.org/cgi-bin/texis/vtx/refworld/rwmain/opendocpdf.pdf? reldoc $=y \& d o c i d=47 e 0 d 26 \mathrm{~d} 2$ (consultado el 13 de noviembre de 2009).

Comité preparatorio en $1996 \mathrm{http} / / \mathrm{www}$.iccnow.org/? $\mathrm{mod}=$ prepcommittee (consultado el 18 de noviembre de 2009).

ONG como Human Rights Watch http://www.iccnow.org/documents/1PrepCmtCommentaryHRW. pdf (consultado el 18 de noviembre de 2009)

International Commission of Jurists http://www.iccnow.org/documents/1PrepCmt3rdPositionP aperICJ.pdf (consultado el 18 de noviembre de 2009).

Artículo 35 del proyecto elaborado por el Comité preparatorio http://www.iccnow.org/documents/ IssuesofAdmissibility.pdf (consultado el 18 de noviembre de 2009). 